\title{
EVALUACIÓN DE MODELOS AGROCLIMÁTICOS DE CÁLCULO DE EVAPOTRANSPIRACIÓN PARA JARDINERÍA. APLICACIÓN EN EL JARDÍN DEL CAMPUS DE VERA DE LA UPV
}

\author{
Clemente Angulo, S. (1), San Bautista, A. (2), Manzano, J. (3), Pascual-Seva, N (4)
}

\begin{abstract}
${ }^{1}$ Graduada en Ingeniería Agroalimentaria y del Medio Rural. ETSIAMN-UPV, Camino de Vera, s/n 46022 Valencia. sanclean22@hotmail.com

${ }^{2}$ Profesor Titular. Universitat Politècnica de València, Centro Valenciano de Estudios sobre el Riego, Departamento de Producción Vegetal, Camino de Vera, s/n 46022 Valencia. asanbau@prv.upv.es

${ }^{3}$ Profesor Contratado Doctor. Universitat Politècnica de València, Centro Valenciano de Estudios sobre el Riego, Departamento Ingeniería Rural y Agroalimentaria, Camino de Vera, s/n 46022 Valencia. juamanju@agf.upv.es

${ }^{4}$ Profesora Ayudante Doctor. Universitat Politècnica de València. Centro Valenciano de Estudios sobre el Riego, Departamento de Producción Vegetal, Camino de Vera, s/n 46022 Valencia. asanbau@prv.upv.es
\end{abstract}

\section{Resumen}

La evapotranspiración (ET) es la combinación de dos procesos separados por los que el agua se pierde a través de la superficie del suelo por evaporación, y por otra parte, mediante transpiración del cultivo. Los factores relacionados con el cultivo y su manejo fueron englobados en la función empírica $\mathrm{Kc}=\mathrm{ET}_{\mathrm{c}} / \mathrm{ET}_{0}$, donde $\mathrm{ET}_{\mathrm{c}}$ es la evapotranspiración de cultivo y $\mathrm{ET}_{0}$ es la evapotranspriación de referencia. Actualmente el método de Penman-Monteith es el recomendado por la FAO y la ASCE (American Society of Civil Engineers) para determinar la $E T_{0}$, requiriendo gran cantidad de parámetros climáticos como radiación, temperatura del aire, humedad atmosférica y velocidad del viento. Existen otros modelos de cálculo (como los propuestos por Hargreaves, Samani y Priestley-Taylor) más sencillos, que son evaluados en este trabajo, utilizando como caso de estudio el jardín de la Universitat Politècnica de València. Adicionalmente los métodos se recalibran, adaptando sus coeficientes empíricos por comparación con la ecuación de Penman-Monteith. Finalmente se realiza un balance de humedad contrastándolo con los valores obtenidos por medido de sondas FDR. Así se ha podido aproximar un valor del coeficiente $\mathrm{Kj}$ para el caso de estudio, que relaciona $E T_{0}$ y $E T_{c}$ en el caso singular de un jardín.

\section{1- Introducción y objetivos}

La evapotranspiración (ET) es la combinación de dos procesos separados por los que el agua se pierde por evaporación a través de la superficie del suelo en contacto directo con el aire exterior, y por otra parte, mediante transpiración del cultivo a través de sus estomas. La evapotranspiración de referencia $\left(E T_{0}\right)$ se define como la tasa de evapotranspiración de un cultivo hipotético de pasto, con una altura de $0,12 \mathrm{~m}$, con una resistencia aerodinámica fija de $70 \mathrm{~s} \mathrm{~m}-1$ y un albedo de 0,23 , es decir, de una superficie extensa de gramíneas verdes de altura uniforme, creciendo activamente y adecuadamente regado (Allen et al., 1989). El concepto de evapotranspiración de referencia se introdujo para estudiar la demanda 
evaporativa de la atmósfera independientemente del tipo de cultivo, de su estadio de desarrollo y de su manejo. Por lo tanto, depende solamente de factores climáticos.

El coeficiente de cultivo es el factor que incluye los efectos propios del cultivo sobre la ET, como pueden ser el área foliar, la altura, el porcentaje de suelo cubierto, etc. Su valor se determina como el cociente entre la evapotranspiración de cultivo y la evapotranspiración de referencia $\left(\mathrm{K}_{\mathrm{c}}=\mathrm{ET}_{\mathrm{c}} / \mathrm{ET}_{0}\right)$ Doorenbos y Pruitt (1975),. Su empleo es válido para un cultivo homogéneo, mientras que para las situaciones típicas en jardinería con diversidad de especies en una parcela se emplea el coeficiente de jardín para describir las necesidades hídricas en las plantas. Según la metodología de Costello et al. (2000), se tienen en cuenta tres coeficientes en función de las especies que componen el jardín $\left(\mathrm{K}_{\mathrm{e}}\right)$, la densidad de plantación $\left(\mathrm{K}_{\mathrm{d}}\right)$ y las condiciones microclimáticas $\left(\mathrm{K}_{\mathrm{m}}\right)$. Por tanto, el coeficiente de jardín será igual al coeficiente de especie por el coeficiente de densidad por el coeficiente de microclima. Con el coeficiente de jardín se pueden estimar, de una forma similar al coeficiente de cultivo, las necesidades de agua a partir de la expresión:

$$
E T_{c}=K_{j} \cdot E T_{0}
$$

La evapotranspiración es uno de los principales componentes del balance hidrológico y su cuantificación es necesaria para la producción agrícola, especialmente en regiones áridas y semiáridas, donde resulta imprescindible para la determinación de las necesidades de agua de los cultivos y por tanto, para aplicar así el riego en cuantía adecuada. La ecuación del balance de humedad de Jensen et al. (1990) es la siguiente:

$$
N_{R}=\left(N_{E T}-N_{E}-N_{P}-\Delta H\right)+N_{P e}
$$

donde:

$\mathrm{N}_{\mathrm{ET}}=$ necesidades de agua para cubrir las pérdidas de agua por ET den el jardín

$\mathrm{N}_{\mathrm{E}}=$ necesidades de agua para cubrir las pérdidas de agua por escorrentía superficial.

$N_{P}=$ necesidades de agua para cubrir las pérdidas de agua por percolación profunda.

$\mathrm{N}_{\mathrm{Pe}}=$ necesidades de agua cubiertas por precipitación efectiva.

$\Delta \mathrm{H}=$ variación del contenido de agua en el suelo.

Para la medida de la evapotranspiración han sido desarrollados muchos procedimientos. Puede ser medida directamente utilizando un lisímetro, que es un gran contenedor abierto por su parte superior que engloba un volumen de suelo cuyo peso puede ser medido con precisión. También puede ser medida indirectamente estableciendo balances de agua en el volumen de suelo enraizado o balance de energía en la superficie foliar; del mismo modo es habitual el empleo de métodos estrictamente micrometeorológico

Actualmente el método de Penman-Monteith (Allen et al., 1998) es el único método recomendado por la FAO y por la ASCE (American Society of Civil Engineers) para determinar la evapotranspiración de referencia con parámetros climáticos de radiación, temperatura del aire, humedad atmosférica y velocidad del viento, ya que se aproxima de manera cercana la $\mathrm{ET}_{0}$ de cualquier localidad evaluada, tiene bases físicas sólidas e incorpora parámetros fisiológicos y aerodinámicos. Pero además, se dispone de una gran variedad de métodos para estimar la $\mathrm{ET}_{0}$ clasificados en función de las variables climáticas requeridas. Algunos métodos basados en temperatura son los de Hargreaves de 1975 y de 1985, y el de Samani (2000), que permiten el cálculo de la $\mathrm{ET}_{0}$ a partir de la temperatura y la radiación solar. Por otra parte, tenemos los métodos basados en radiación como el de Priestley-Taylor (1972) y el de Makkink (1957), que se basan en la alta correlación existente entre la evapotranspiración y la radiación solar. 
El principal inconveniente que presenta la ecuación de Penman-Monteith es la gran cantidad de variables requeridas, ya que los registros de entrada de estas variables son, a veces, de calidad cuestionable, incompletos o no disponibles para un lugar determinado, especialmente en los países en desarrollo.

Por ello, el principal objetivo de este trabajo es evaluar modelos agroclimáticos de cálculo de la evapotranspiración de referencia más sencillos, utilizando como criterio de comparación la ecuación de Penman-Monteith. Además, también se pretende obtener un balance de humedad a partir de variables climáticas y parámetros de riego, con el fin de establecer un coeficiente de jardín en el jardín de la Universitat Politècnica de València (U.P.V.) que relacione la evapotranspiración de referencia y la evapotranspiración del cultivo.

\section{2- Materiales y métodos}

El estudio se realiza para el período de años de 2010 a 2013 a partir de los datos climáticos diarios y mensuales de radiación extraterrestre, temperatura, humedad relativa y velocidad del viento, obtenidos de la estación meteorológica de la Universidad Politécnica de Valencia. La estación se encuentra a una latitud de $39^{\circ} 40^{\prime} \mathrm{E}$, a una longitud de $0^{\circ} 22^{\prime}$ y a una altitud de $11 \mathrm{~m}$. Se midió la radiación extraterrestre $(\mathrm{Ra})$ máxima, mínima y media, en $\mathrm{wm}^{-2}$, temperatura máxima, mínima y media, en ${ }^{\circ} \mathrm{C}$; humedad relativa máxima, mínima y media, en $\%$ y la velocidad del viento $\left(\mathrm{u}_{2}\right)$, en $\mathrm{m} \mathrm{s}^{-1}$, considerando la altura del anemómetro de 6,6 $\mathrm{m}$.

Con los datos climáticos se calcula la $\mathrm{ET}_{0}$ utilizando los métodos de Penman-Monteith (Allen et al., 1998), Hargreaves (1975), Hargreaves (1985), Samani (2000) y Priestley-Taylor (1972), siendo la formulación de los métodos la expuesta en la Tabla 1

Tabla 1. Modelos propuestos para el cálculo de la evapotranspiración.

\begin{tabular}{|c|c|c|}
\hline Método & Referencia & Ecuación \\
\hline Método FAO/ASCE & Allen et al. ( 1998) & $0,408 \Delta\left(R_{n}-G\right)+\gamma \frac{C_{n}}{T+273} u_{2}\left(e_{s}-e_{a}\right)$ \\
\hline & & $\Delta+\gamma\left(1+C_{d} u_{2}\right)$ \\
\hline \multirow{6}{*}{$\begin{array}{l}\text { Métodos de } \\
\text { Temperatura }\end{array}$} & Hargreaves (1975) & $\mathrm{ET}_{\text {Har1 }}=\mathrm{A} \cdot \mathrm{R}_{\mathrm{s}} \cdot\left(\mathrm{T}_{\mathrm{m}}+17,8\right)$ \\
\hline & \multirow{2}{*}{$\begin{array}{l}\text { (Hargreaves 1) } \\
\text { Hargreaves (1985) }\end{array}$} & $\mathrm{ET}_{\text {Har1 }}=\mathrm{A} \cdot \mathrm{R}_{\mathrm{s}} \cdot\left(\mathrm{T}_{\mathrm{m}}+\mathrm{B}\right)$ \\
\hline & & $\mathrm{ET}_{\text {Har2 }}=\mathrm{A} \cdot \mathrm{R}_{\mathrm{a}} \cdot\left(\mathrm{T}_{\mathrm{m}}+17,8\right) \cdot \Delta \mathrm{T}^{1 / 2}$ \\
\hline & \multirow[t]{2}{*}{ (Hargreaves 2) } & $\mathrm{ET}_{\text {Har2 }}=\mathrm{A} \cdot \mathrm{R}_{\mathrm{a}} \cdot\left(\mathrm{T}_{\mathrm{m}}+\mathrm{B}\right) \cdot \Delta \mathrm{T}^{1 / 2}$ \\
\hline & & $\mathrm{ET}_{\text {Har2 }}=\mathrm{A} \cdot \mathrm{R}_{\mathrm{a}} \cdot\left(\mathrm{T}_{\mathrm{m}}+\mathrm{B}\right) \cdot \Delta \mathrm{T}^{\mathrm{C}}$ \\
\hline & Samani (2000) & $\begin{array}{c}\text { ET Sam }=0,0135 \cdot\left(\mathrm{C} \cdot \mathrm{R}_{\mathrm{a}} \cdot \Delta \mathrm{T}^{1 / 2}\right) \cdot\left(\mathrm{T}_{\mathrm{m}}+17,8\right) \\
\mathrm{C}=\left(\mathrm{a}_{1} \cdot \Delta \mathrm{T}^{2}\right)+\left(\mathrm{a}_{2} \cdot \Delta \mathrm{T}\right)+\mathrm{a}_{3}\end{array}$ \\
\hline \multirow[t]{2}{*}{ Método de Radiación } & \multirow[t]{2}{*}{$\begin{array}{l}\text { Priestley \& Taylor } \\
(1972)\end{array}$} & $\lambda \mathrm{ET}_{\text {Priest }}=\alpha \cdot\left(\frac{\Delta}{\Delta+\gamma}\right) \cdot\left(\mathrm{R}_{\mathrm{n}}-\mathrm{G}\right)$ \\
\hline & & $\lambda \mathrm{ET}_{\text {Priest }}=\alpha \cdot\left(\frac{\Delta}{\Delta+\mathrm{\gamma}}\right)^{\mathrm{B}} \cdot\left(\mathrm{R}_{\mathrm{n}}-\mathrm{G}\right)^{\mathrm{C}}$ \\
\hline \multicolumn{2}{|c|}{$\begin{array}{l}E T_{0}=\text { evapotranspiración de referencia } \\
\mathrm{R}_{\mathrm{n}}=\text { radiación neta en la superficie del cultivo } \\
\mathrm{G}=\text { flujo del calor del suelo } \\
\mathrm{T}=\text { temperatura media del aire a } 2 \text { m de altura } \\
\mathrm{U}_{2}=\text { velocidad del viento a } 2 \text { m de altura } \\
\mathrm{e}_{\mathrm{s}}=\text { presión de vapor de saturación } \\
\mathrm{e}_{\mathrm{a}}=\text { presión real de vapor } \\
\mathrm{e}_{\mathrm{s}} \text { - e } \mathrm{e}_{\mathrm{a}}=\text { déficit de presión de vapor } \\
\Delta=\text { pendiente de la curva de presión de vapor } \\
\mathrm{V}=\text { constante psicrométrica }\end{array}$} & $\begin{array}{l}\mathrm{R}_{\mathrm{s}}=\text { radiación solar total } \\
\mathrm{T}_{\mathrm{m}}=\text { temperatura media diaria del aire } \\
\Delta \mathrm{T}=\text { diferencia entre la temperatura máxima } \\
\text { diaria y la temperatura mínima diaria } \\
\mathrm{R}_{\mathrm{a}}=\text { radiación extraterrestre } \\
\alpha=\text { albedo o coeficiente de reflexión }=1,26 \\
\Delta=\text { Pendiente de la curva de presión de } \\
\text { saturación de vapor } \\
\lambda=\text { calor latente } \\
P=\text { presión atmosférica } \\
\mathrm{a}_{1}, \mathrm{a}_{2}, \mathrm{a}_{3}=\text { coeficientes de ec. Samani }\end{array}$ \\
\hline
\end{tabular}


Una vez calculada la $E T_{0}$ con dichos métodos, aplicando valores diarios de las variables climáticas intervinientes en cada método y también valores medios para cada mes, se proponen la adaptación de los modelos con modificaciones en sus principales coeficientes. Para ello, se estiman los coeficientes de ajuste mediante una recalibración entre los valores de la $\mathrm{ET}_{0}$ de Penman-Monteith y las variables climáticas intervinientes en cada modelo, aplicando un ajuste no lineal para cada modelo estudiado. El programa utilizado para realizar el análisis estadístico fue el Statgraphics Centurion versión 16.1.17.

Como último paso y con el objeto de obtener un valor práctico del coeficiente de jardín para el caso de estudio, se calcula el balance de humedad del suelo obtenido como la diferencia que se produce entre las entradas de agua (por precipitación o riego) y las salidas (por evapotranspiración, despreciando las salidas de agua por escorrentía superficial y por percolación profunda).

Para la de medición de la humedad utilizada en el suelo del jardín de la U.P.V. se empleó una sonda FDR (Frequency Domain Refletometry) a tres profundidades (10, 30 y 50 $\mathrm{cm}$ ), obteniéndose los siguientes valores de humedad para el período del 15 de julio al 31 de diciembre de 2013.

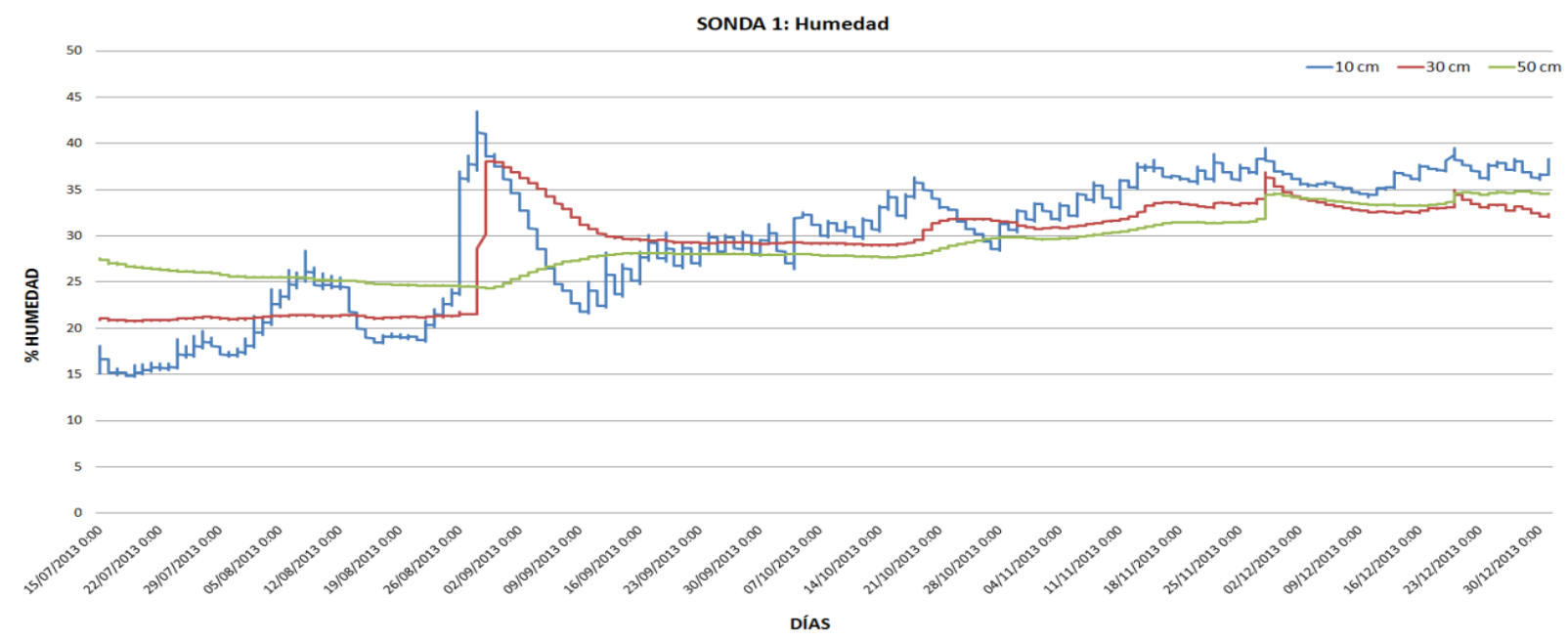

Figura 1. Humedad en suelo medida por la sonda FDR a tres profundidades.

Para conocer el volumen de riego aportado se realizó un ensayo de pluviometría sobre la zona de influencia de la sonda, para un día sin viento. Se dispusieron 49 pluviómetros separados entre sí a $30 \mathrm{~cm}$ (matriz 7x7) y se regó la zona reproduciendo un riego ordinario de15 minutos de duración. A partir de dicho ensayo se obtuvo el volumen de pluviometría y la dosis de riego aplicada en el jardín. Como método de riego se utilizó el riego por aspersión, con un marco de aspersión de 12x12. Los aspersores son modelo tipo SUPERPRO de Safe Rain. La especie de césped en la zona de nuestro ensayo fue una mezcla básica de cespitosas compuesta por Cynodon dactylon, Festuca arundinacea, Penisetum clandestinum y Poa annua.

Por otra parte, a partir de los datos de precipitación diarios obtenidos de la estación meteorológica de la U.P.V. se calculó la precipitación efectiva, como aquella fracción de la precipitación total que es aprovechada por las plantas, utilizando la siguiente expresión de Dastane (1974): $\mathrm{Pe}=0,75 \times \mathrm{PT}-5$. A partir de la dosis de riego, de la precipitación efectiva y de la $\mathrm{ET}_{0}$ de Penman-Monteith, se obtuvo el balance de humedad en el jardín. 
Se comparó el contenido de humedad obtenido mediante el balance y el contenido de humedad medido mediante sondas FDR y ambos contenidos de humedad se ajustaron multiplicando por un valor las necesidades de agua para cubrir las pérdidas de agua por ET, aproximando de esta forma el coeficiente de jardín.

\section{3- Resultados y discusión}

En la tabla 2 se muestran las correlaciones entre las distintas variables climáticas utilizadas en este estudio y los valores estimados de la $\mathrm{ET}_{0}$ mensual y diaria utilizando los cuatro métodos alternativos con los valores originales de sus constantes.

Tabla 2. Matriz de correlación entre las distintas variables climáticas y los valores estimados de $\mathrm{ET}_{0}$ mensual y diario $\left({ }^{*},{ }^{* *}\right.$ : nivel de significación, $\mathrm{P}<0,05$ y $\mathrm{P}<0,01$, respectivamente).

\begin{tabular}{|c|c|c|c|c|c|c|c|c|c|c|c|}
\hline \multicolumn{12}{|c|}{ Matiz valores mensuales } \\
\hline Variable & $\mathbf{R a}_{\mathbf{a}}$ & Rs & HR & $\mathbf{u}^{2}$ & $T_{m}$ & $\Delta \mathbf{T}$ & ETPriest & ET $_{\text {Har1 }}$ & $\mathrm{ET}_{\text {Har2 }}$ & ETsam & ETPen \\
\hline $\mathbf{R}_{\mathbf{a}}$ & 1 & & & & & & & & & & \\
\hline $\mathbf{R}_{\mathrm{s}}$ & $0,97^{* *}$ & 1 & & & & & & & & & \\
\hline HR & $0,57^{\star *}$ & $0,53^{* *}$ & 1 & & & & & & & & \\
\hline$u^{2}$ & 0,10 & 0,05 & $-0,30$ & 1 & & & & & & & \\
\hline$T_{m}$ & $0,74^{* *}$ & $0,76^{* *}$ & 0,72 & $-0,17$ & 1 & & & & & & \\
\hline$\Delta T$ & $-0,08$ & 0,03 & $-0,48$ & $-0,03$ & $-0,37^{* *}$ & 1 & & & & & \\
\hline ET Priest & $0,98^{\star *}$ & $0,97^{* *}$ & $0,65^{* *}$ & 0,01 & $0,85^{\star *}$ & $-0,14$ & 1 & & & & \\
\hline ET & $0,95^{\star \star}$ & $0,97^{* *}$ & $0,63^{* *}$ & $-0,02$ & $0,88^{* *}$ & $-0,11$ & $0,99^{* \star}$ & 1 & & & \\
\hline $\mathrm{ET}_{\text {Har2 }}$ & $0,98^{* *}$ & $0,97^{* *}$ & $0,62^{* *}$ & 0,01 & $0,84^{* *}$ & $-0,10$ & $0,99^{* *}$ & $0,99^{* *}$ & 1 & & \\
\hline ETsam & $0,98^{* *}$ & $0,96^{* *}$ & $0,64^{* *}$ & 0,03 & $0,86^{* *}$ & $-0,17$ & $0,99^{* *}$ & $0,98^{\star *}$ & $0,64^{* *}$ & 1 & \\
\hline $\mathrm{ET}_{\text {Penm }}$ & $0,96^{* *}$ & $0,96^{* *}$ & $0,51^{* *}$ & 0,21 & $0,81^{* *}$ & $-0,11$ & $0,97^{* *}$ & $0,97^{* *}$ & $0,97^{* *}$ & $0,97^{\star \star}$ & 1 \\
\hline \multicolumn{12}{|c|}{ Matriz valores diarios } \\
\hline Variable & $\mathbf{R a}_{\mathbf{a}}$ & Rs & HR & $\mathbf{u}^{2}$ & $\mathrm{~T}_{\mathrm{m}}$ & $\Delta \mathbf{T}$ & ET Priest & ET $_{\text {Harg1 }}$ & ET $_{\text {Harg2 }}$ & $\mathrm{ET}_{\mathrm{sam}}$ & ETPen \\
\hline $\mathbf{R}_{\mathbf{a}}$ & 1 & & & & & & & & & & \\
\hline $\mathbf{R}_{\mathrm{s}}$ & $0,77^{* \star}$ & 1 & & & & & & & & & \\
\hline HR & $0,29^{\star *}$ & $0,07^{* \star}$ & 1 & & & & & & & & \\
\hline $\mathbf{u}^{2}$ & 0,05 & $-0,03$ & $-0,31^{* *}$ & 1 & & & & & & & \\
\hline$T_{m}$ & $0,70^{* *}$ & $0,59^{* *}$ & $0,31^{* *}$ & $-0,03$ & 1 & & & & & & \\
\hline$\Delta \mathbf{T}$ & $-0,03$ & $0,20^{* *}$ & $-0,29^{* *}$ & $-0,20^{* *}$ & $-0,02$ & 1 & & & & & \\
\hline$\overline{E T_{\text {Priest }}}$ & $0,92^{\star *}$ & $0,87^{* *}$ & $0,30^{\star *}$ & $-0,01$ & $0,79^{* *}$ & 0,02 & 1 & & & & \\
\hline $\mathrm{ET}_{\text {Har1 }}$ & $0,81^{* *}$ & $0,92^{* *}$ & $0,15^{\star *}$ & $-0,01$ & $0,76^{* \star}$ & $0,18^{* *}$ & $0,91^{* *}$ & 1 & & & \\
\hline $\mathrm{ET}_{\text {Har2 }}$ & $0,91^{* *}$ & $0,77^{* \star}$ & $0,22^{\star *}$ & $-0,02$ & $0,79^{\star *}$ & $0,23^{* *}$ & $0,91^{* *}$ & $0,86^{* *}$ & 1 & & \\
\hline ETsam & $0,94^{* *}$ & $0,75^{* \star}$ & $0,28^{* *}$ & 0,02 & $0,83^{* *}$ & $0,07^{* *}$ & $0,92^{* *}$ & $0,85^{\star *}$ & $0,95^{* *}$ & 1 & \\
\hline $\mathrm{ET}_{\text {Penm }}$ & $0,83^{\star *}$ & $0,80^{* *}$ & $-0,01$ & $0,31^{* *}$ & $0,72^{\star *}$ & 0,04 & $0,89^{* *}$ & $0,82^{* *}$ & $0,82^{\star *}$ & $0,83^{* *}$ & 1 \\
\hline
\end{tabular}

Para los valores mensuales se puede observar que se han obtenido correlaciones muy positivas y estadísticamente significativas entre la variable $R_{s}$ y entre los valores de $E T_{\text {Priest, }}$,

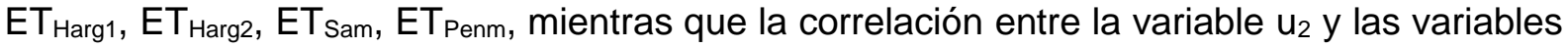
$H R, T_{m}, \Delta T$ es negativa. Para los valores diarios se puede ver que también se han obtenido correlaciones muy positivas y estadísticamente significativas entre la variable $R_{s}$ y entre los valores diarios de $\mathrm{ET}_{\text {Priest }}, \mathrm{ET}_{\text {Harg1 }}, \mathrm{ET}_{\text {Harg2, }} \mathrm{ET}_{\text {Sam, }}, \mathrm{ET}_{\text {Penm. }}$. Por último, entre la variable $\Delta \mathrm{T}$ y las variables $\mathrm{HR}$ y u $\mathrm{u}_{2}$ se obtienen correlaciones negativas y estadísticamente significativas. 
A modo de ejemplo se muestra en la siguientes figuras los valores de la evapotranspiración mensual (Figura 2) y diaria (Figura 3) para el método de Hargreaves (1975) comparado con el método de Penman-Monteith. Para obtener una comparación más cuantitativa, las correlaciones estimadas entre los cuatro métodos alternativos y el método de Penman-Monteith fueron analizadas usando una ecuación de regresión lineal. $Y=m X+c$, donde $\mathrm{Y}$ representa la ET calculada con la Ecuación de Penman-Monteith y $\mathrm{X}$ es la ET estimada a partir de los cuatro métodos mencionados anteriormente, siendo $m$ y c constantes que representan la pendiente y la intersección de la ecuación de regresión, respectivamente
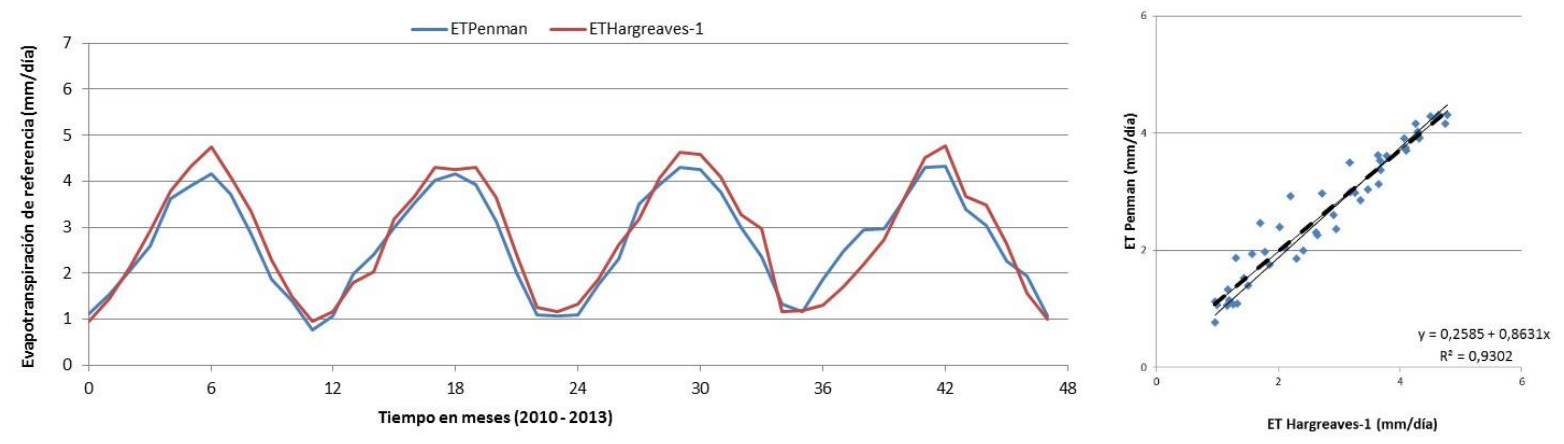

Figura 2. Gráfico de la evapotranspiración de referencia mensual calculada con el método de Penman-Monteith y el método de Hargreaves11 (Izq.) y su comparación mediante regresión lineal (dcha).
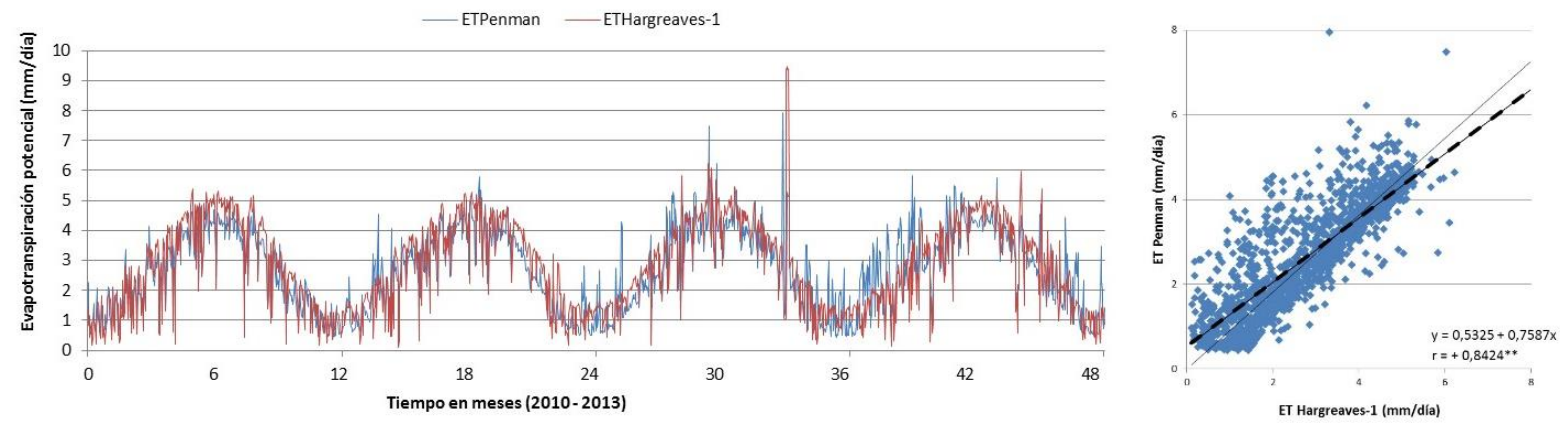

Figura 3. Gráfico de la evapotranspiración de referencia diaria calculada con el método de Penman-Monteith y el método de Hargreaves-1 (Izq.) y su comparación mediante regresión lineal (dcha).

En relación a la ET mensual, y respecto a los valores del coeficiente de correlación $r$, el método de Priestley-Taylor tiene el más alto con $r=0,9730$ y el método Hargreaves-1, tiene el valor más bajo con $r=0,9691$. En cuanto a la pendiente de las ecuaciones de regresión, el método de Hargreaves- 1 resultó con una pendiente cercana a la unidad $(0,8631)$. Respecto a la ET diaria, el método de Priestley-Taylor tiene el valor del coeficiente de correlación más alto con 0,8862 y el método de Hargreaves-2 presenta el valor más bajo con $r=0,8191$. Finalmente la pendiente de las ecuaciones de regresión, el método de Samani tiene la pendiente más cercana a la unidad $(0,7918)$.

En la tabla siguiente se muestran los valores de los parámetros originales y los valores adoptados después de la recalibración para cada ecuación alternativa y el nivel de significación. Para valores mensuales de ET, se puede comprobar que el método que presenta el coeficiente de determinación $R^{2}$ más elevado es el de Hargreaves-2 (de 0,9509), utilizando la recalibración de los parámetros $A, B$ y $C$ de la ecuación. Para valores diarios de 
ET, se observa que el método con $R^{2}$ más elevada es el de Priestley-Taylor (de 0,7809) utilizando la recalibración de los parámetros $\alpha$, B y C de la ecuación.

Tabla 3. Comparación de los valores de los parámetros de las ecuaciones antes y después de la recalibración, para valores mensuales de evapotranspiración

\begin{tabular}{|c|c|c|c|c|c|}
\hline \multicolumn{6}{|c|}{ Valores mensuales } \\
\hline \multirow[t]{2}{*}{ Referencia } & \multirow[t]{2}{*}{ Ecuación } & \multicolumn{4}{|c|}{ Valores de los parámetros } \\
\hline & & Original & Recalibrado & $\mathbf{R}^{2}$ & Probabilidad \\
\hline \multirow[t]{2}{*}{ Har1(1975) } & $\mathrm{ET}=\mathrm{A} \cdot \mathrm{R}_{\mathrm{s}} \cdot\left(\mathrm{T}_{\mathrm{m}}+17,8\right)$ & $A=0,0135$ & $A=0,0127$ & 0,9303 & $<0,00001$ \\
\hline & $E T=A \cdot R_{s} \cdot\left(T_{m}+B\right)$ & $\begin{array}{l}A=0,0135 \\
B=17,8\end{array}$ & $\begin{array}{l}A=0,0076 \\
B=43,7209\end{array}$ & 0,9466 & $<0,00001$ \\
\hline \multirow[t]{3}{*}{ Har2(1985) } & $\mathrm{ET}=\mathrm{A} \cdot \mathrm{R}_{\mathrm{a}} \cdot\left(\mathrm{T}_{\mathrm{m}}+17,8\right) \cdot \Delta \mathrm{T}^{1 / 2}$ & $A=0,0023$ & $A=0,0020$ & 0,9413 & $<0,00001$ \\
\hline & $\mathrm{ET}=\mathrm{A} \cdot \mathrm{R}_{\mathrm{a}} \cdot\left(\mathrm{T}_{\mathrm{m}}+\mathrm{B}\right) \cdot \Delta \mathrm{T}^{1 / 2}$ & $\begin{array}{l}A=0,0023 \\
B=17,8\end{array}$ & $\begin{array}{l}A=0,0015 \\
B=30,0078\end{array}$ & 0,9472 & $<0,00001$ \\
\hline & $\mathrm{ET}=\mathrm{A} \cdot \mathrm{R}_{\mathrm{a}} \cdot\left(\mathrm{T}_{\mathrm{m}}+\mathrm{B}\right) \cdot \Delta \mathrm{T}^{\mathrm{C}}$ & $\begin{array}{l}A=0,0023 \\
B=17,8 \\
C=0,5\end{array}$ & $\begin{array}{l}A=0,0026 \\
B=38,8791 \\
C=0,1833\end{array}$ & 0,9509 & $<0,00001$ \\
\hline Sam. (2000) & $\begin{array}{l}\mathrm{ET}=0,0135 \cdot\left(\mathrm{C} \cdot \mathrm{R}_{\mathrm{a}} \cdot \Delta \mathrm{T}^{1 / 2}\right) \cdot\left(\mathrm{T}_{\mathrm{m}}+\right. \\
17,8) \\
\mathrm{C}=\left(\mathrm{a}_{1} \cdot \Delta \mathrm{T}^{2}\right)+\left(\mathrm{a}_{2} \cdot \Delta \mathrm{T}\right)+\mathrm{a}_{3}\end{array}$ & $\begin{array}{l}a_{1}=0,00185 \\
a_{2}=-0,0433 \\
a_{3}=0,4023\end{array}$ & $\begin{array}{l}a_{1}=0,00094 \\
a_{2}=-0,0181 \\
a_{3}=0,2362\end{array}$ & 0,9417 & $<0,00001$ \\
\hline \multirow[t]{2}{*}{ Priest (1972) } & $\lambda \mathrm{ET}=\alpha \cdot\left(\frac{\Delta}{\Delta+y}\right) \cdot\left(\mathrm{R}_{\mathrm{n}}-\mathrm{G}\right)$ & $\alpha=1,26$ & $\alpha=0,5043$ & 0,7875 & $<0,00001$ \\
\hline & $\lambda E T=\alpha \cdot\left(\frac{\Delta}{\Delta+\gamma}\right)^{B} \cdot\left(R_{n}-G\right)^{C}$ & $\begin{array}{l}\alpha=1,26 \\
\mathrm{~B}=1 \\
\mathrm{C}=1\end{array}$ & $\begin{array}{l}\alpha=0,9945 \\
B=0,5123 \\
C=0,6269\end{array}$ & 0,9410 & $<0,00001$ \\
\hline \multicolumn{6}{|c|}{ Valores diarios } \\
\hline \multirow[t]{2}{*}{ Referencia } & Ecuación & \multicolumn{4}{|c|}{ Valores de los parámetros } \\
\hline & & Original & Recalibrado & $\mathbf{R}^{\mathbf{2}}$ & Probabilidad \\
\hline \multirow[t]{2}{*}{ Har1(1975) } & $\mathrm{ET}=\mathrm{A} \cdot \mathrm{Rs} \cdot(\mathrm{Tm}+17,8)$ & $A=0,0135$ & $A=0,0123$ & 0,6936 & $<0,00001$ \\
\hline & $\mathrm{ET}=\mathrm{A} \cdot \mathrm{Rs} \cdot(\mathrm{Tm}+\mathrm{B})$ & $\begin{array}{l}A=0,0135 \\
B=17,8\end{array}$ & $\begin{array}{l}A=0,0108 \\
B=23,0782\end{array}$ & 0,6948 & $<0,00001$ \\
\hline \multirow[t]{3}{*}{ Har2(1985) } & $\mathrm{ET}=\mathrm{A} \cdot \mathrm{Ra} \cdot(\mathrm{Tm}+17,8) \cdot \Delta \mathrm{T} 1 / 2$ & $A=0,0023$ & $A=0,0020$ & 0,6753 & $<0,00001$ \\
\hline & $\mathrm{ET}=\mathrm{A} \cdot \mathrm{Ra} \cdot(\mathrm{Tm}+\mathrm{B}) \cdot \Delta \mathrm{T} 1 / 2$ & $\begin{array}{l}A=0,0023 \\
B=17,8\end{array}$ & $\begin{array}{l}A=0,0017 \\
B=26,0616\end{array}$ & 0,6777 & $<0,00001$ \\
\hline & $\mathrm{ET}=\mathrm{A} \cdot \mathrm{Ra} \cdot(\mathrm{Tm}+\mathrm{B}) \cdot \Delta \mathrm{TC}$ & $\begin{array}{l}A=0,0023 \\
B=17,8 \\
C=0,5\end{array}$ & $\begin{array}{l}A=0,0036 \\
B=27,7911 \\
C=0,1291\end{array}$ & 0,7312 & $<0,00001$ \\
\hline Sam. (2000) & $\begin{array}{l}\mathrm{ET}=0,0135 \cdot(\mathrm{C} \cdot \mathrm{Ra} \cdot \Delta \mathrm{T} 1 / 2) \cdot(\mathrm{Tm} \\
+17,8) \\
\mathrm{C}=(\mathrm{a} 1 \cdot \Delta \mathrm{T} 2)+(\mathrm{a} 2 \cdot \Delta \mathrm{T})+\mathrm{a} 3\end{array}$ & $\begin{array}{l}a 1=0,00185 \\
a 2=-0,0433 \\
a 3=0,4023\end{array}$ & $\begin{array}{l}a 1=0,00029 \\
a 2=-0,0117 \\
a 3=0,2330\end{array}$ & 0,7241 & $<0,00001$ \\
\hline \multirow[t]{2}{*}{ Priest (1972) } & $\lambda E T=\alpha \cdot\left(\frac{\Delta}{\Delta+\gamma}\right) \cdot(R n-G)$ & $\alpha=1,26$ & $\alpha=0,5008$ & 0,6781 & $<0,00001$ \\
\hline & $\lambda E T=\alpha \cdot\left(\frac{\Delta}{\Delta+\gamma}\right)^{B} \cdot(R n-G) C$ & $\begin{array}{l}\alpha=1,26 \\
B=1 \\
C=1\end{array}$ & $\begin{array}{l}a=1,2508 \\
B=0,9304 \\
C=0,5979\end{array}$ & 0,7809 & $<0,00001$ \\
\hline
\end{tabular}

Utilizando la recalibración de los valores constantes de $\mathrm{A}$ y $\mathrm{B}$ en el método de Hargreaves-1, mejora el coeficiente de correlación de 0,9691 a 0,9729. En cuanto al método de Hargreaves-2, se observa que con los valores originales de las constantes funciona bastante bien, y que presenta un coeficiente de correlación mayor que con el método anterior 
(de 0,97). Utilizando los valores A, B y C recalibrados el método mejora ligeramente. La ecuación de Samani también funciona bastante bien con los valores originales de las constantes y tras la recalibración de sus coeficientes, los resultados permanecen prácticamente invariables. En cuanto al método de Priestley -Taylor, el valor de $\alpha=1,26$ resulta demasiado elevado para la región. Se observa que se logra una importante mejora cuando se utilizan las constantes $\alpha, \mathrm{B}$ y $\mathrm{C}$ recalibradas. Si bien, el coeficiente de correlación se mantiene.

En relación a la evapotranspiración diaria, se obtienen coeficientes de correlación menores que con datos mensuales de $\mathrm{ET}_{0}$, aunque los resultados también son estadísticamente significativos. El método de Hargreaves-1 funciona bastante bien con los valores originales de las constantes y tras la recalibración, los resultados prácticamente no varían. El método de Hargreaves-2 con los valores originales de sus constantes es el que presenta el valor más bajo de coeficiente de correlación con $r=0,819$. Tras la recalibración, se comprueba que el método mejora su coeficiente de correlación de 0,819 a 0,851, cuando se utilizan los valores $A, B$ y $C$ recalibrados. El método de Samani, con los valores originales de sus constantes, es el que presenta la pendiente de la ecuación más cercana a la unidad $(0,7918)$. Tras la recalibración, se constata que el método mejora ligeramente (su coeficiente de correlación pasa de 0,8316 a 0,8525). En relación al método de Priestley-Taylor, con los valores originales de sus constantes es el método que presenta el valor de coeficiente de correlación más alto $(0,8862)$ para valores de ET diarios, pero al igual que en el caso mensual, se observa que el valor de $\alpha=1,26$ de la ecuación es demasiado alto para la región. Por último, se logra una importante mejora en el método de Priestley-Taylor cuando se utilizan las constantes $\alpha$, B y $\mathrm{C}$ recalibradas; si bien el coeficiente de correlación se mantiene.

En la última fase se procede a estimar el coeficiente de jardín. A partir del experimento de pluviometría comentado, se obtuvo una dosis de riego de $1,86 \mathrm{~mm}$. Con esta dosis de riego, la $\mathrm{ET}_{0}$ de Penman-Monteith y la precipitación efectiva calculada, se obtuvo el balance de humedad en el suelo. En la siguiente figura se representa el contenido de humedad calculado mediante el balance de humedad y contenido de humedad en el suelo medido mediante sondas FDR. Para ligar ambos conjuntos de valores, la $\mathrm{ET}_{0}$ de Penman-Monteith en la ecuación del balance ha sido ajustada con un factor de 0,4 , asimilable al coeficiente de jardín y comparable al obtenido en otros trabajos.

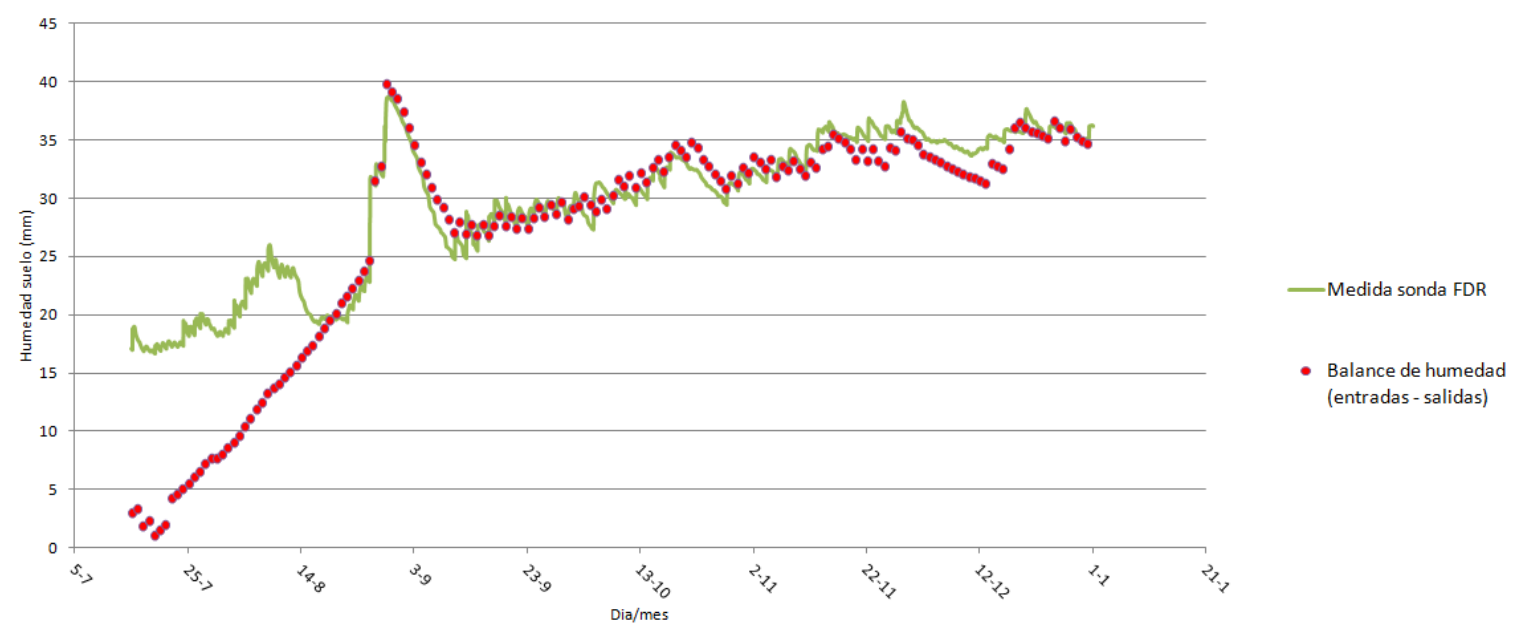

Figura 1. Gráfico del contenido de humedad en el suelo medido mediante sondas FDR y el contenido de humedad calculado mediante el balance de entradas y salidas de agua. 


\section{4- Conclusiones}

Se ha obtenido una correlación muy positiva y estadísticamente significativa entre la radiación solar y la evapotranspiración calculada con los métodos de Hargreaves y PriestleyTaylor, constatándose que dicha variable es el principal factor que interviene en las ecuaciones de ambos métodos. Se ha obtenido una correlación negativa y estadísticamente significativa entre la velocidad del viento $\left(u_{2}\right)$ y la diferencia entre las temperaturas máxima y mínima diarias $(\Delta T)$, verificandose que en las zonas costeras la elevada $u_{2}$ tiende a reducir la temperatura.

Se observa que con datos mensuales de $\mathrm{ET}_{0}$ se obtienen coeficientes de correlación mucho más elevados que con datos diarios, si bien, los modelos matemáticos en ambos casos han resultado ser estadísticamente significativos.

Con datos mensuales de $\mathrm{ET}_{0}$, de los modelos matemáticos estudiados en este trabajo, el mejor resultado se ha obtenido con el método de Hargreaves (1985) con la siguiente modificación de sus coeficientes: $E T_{0}=0,0026 \cdot R_{a} \cdot\left(T_{m}+38,8791\right) \cdot \Delta T^{0.1833}$; con $R^{2}=0,9509$.

Para datos diarios de $\mathrm{ET}_{0}$, de los modelos matemáticos estudiados en este trabajo, el mejor resultado se ha obtenido con el método Priestley-Taylor con la siguiente modificación de sus coeficientes: $\lambda E T=1,2508 \cdot\left(\frac{\Delta}{\Delta+\gamma}\right)^{0,9304}(\mathrm{Rn}-\mathrm{G})^{0.5979} ;$ con $\mathrm{R}^{2}=0,7809$.

Por otra parte, se observa una buena correlación entre los niveles de humedad en el suelo y las pérdidas de agua por evapotranspiración calculadas con el método de PenmanMonteith. El valor de Kj que se puede proponer según nuestro experimento de campo sería de 0,4 para el caso de estudio.

Por último, decir que los modelos estudiados podrían ser utilizados como alternativa al método de Penman-Monteith para las condiciones climáticas de la ciudad de Valencia.

\section{5.- Agradecimientos}

Este trabajo ha sido financiado por la ayuda SP20120823 del Programa de Apoyo a la Investigación y Desarrollo (PAID-06-12) del Vicerrectorado de Investigación de la Universitat Politècnica de València a través del proyecto "Estrategias para el ahorro de agua y energía en jardinería Mediterránea. Comparación de las necesidades de riego determinadas con modelos agro-hidrológicos y sondas de humedad de suelo e integración en la gestión hidráulica".

\section{6.- Bibliografía}

Allen, R.G., Jensen, M.E, Wright, J.L. and Burman, R.D. (1989). Operational estimates of reference evapotranspiration. Agronomy Journal, 81: 650-662.

Allen, R.G., Pereira, L.S., Raes, D. and Smith, M. (1998). Crop Evapotranspiration. FAO Irrigation and Drainage Paper No. 56. Rome. 300 pp.

Costello, L. R. and Jones, K. S. (2000). Water use classification of landscape species. A guide to the water needs of landscape plants. California Department of Water Resources. University of California Cooperative Extension. 150 pp.

Dasante, N.G. (1974). Effective rainfall in irrigated agriculture. FAO Irrigation and Drainage Paper No. 25. Rome. 62 pp. 
Doorenbos, J. and Pruitt, W.O. (1977). Crop water requirements. FAO Estudio de Riego y Drenaje No. 24, (rev.). Organización de las Naciones Unidas para la Agricultura y la Alimentación. Roma. 144 pp.Hargreaves, G.H. (1975). Moisture availability and crop production. Transaction of the American Society of Civil Engineers, 18 (5): 980-984.

Hargreaves, G.L.; Hargreaves, G.H. and Riley, J.P. (1985). Agricultural benefits for Senegal River Basin. Journal of Irrigation and Drainage Engineering, 111: 113-124.

Hargreaves, G.H. and Samani, Z.A. (1985). Reference Crop Evapotranspiration from Temperature. Applied Engineering in Agriculture, 1 (2): 96-99.

Jensen, M.E.; Burman, R.D. and Allen, R.G. (1990). Evapotranspiration and Irrigation Water Requirements. ASCE Manuals and Reports on Engineering Practices No. 70, American Society of Civil Engineers. New York, Estados Unidos de América. 360 pp.

Makkink, G.F. (1957). Testing the Penman formula by means of lysimeters. Journal of the Institute of Water Engineers, 11 (3): 277-288.

Priestley, C.H.B. and Taylor, R.J. (1972). On the assessment of surface heat flux and evaporation using large scale parameters. Monthly Weather Review, 100: 81-92.

Samani, Z.A. (2000). Estimating solar radiation and evapotranspiration using minimum climatological data. Journal of Irrigation and Drainage Engineering, 126 (4): 265-267. 